\title{
Investigation of plasma hydrogenation and trapping mechanism for layer transfer
}

\author{
Peng Chen and Paul K. Chu ${ }^{\text {a) }}$ \\ Department of Physics and Materials Science, City University of Hong Kong, Tat Chee Avenue, \\ Kowloon, Hong Kong \\ T. Höchbauer, J.-K. Lee, and M. Nastasi \\ Los Alamos National Laboratory, Los Alamos, New Mexico, 87545 \\ D. Buca and S. Mantl \\ Institut für Schicht- und Ionentechnik, Forschungszentrum Jülich GmbH, D-52425 Jülich, Germany \\ R. Loo and M. Caymax \\ IMEC, Kapeldreef 75, B - 3001 Leuven, Belgium \\ T. Alford and J. W. Mayer \\ Department of Chemical and Materials Engineering, Arizona State University, Tempe, Arizona 85287 \\ N. David Theodore \\ Advanced Products R\&D Lab., Motorola Inc., 2100 East Elliot Road, Tempe, Arizona 85284
}

M. Cai, B. Schmidt, and S. S. Lau

University of California at San Diego, San Diego, California, 92093

(Received 13 May 2004; accepted 15 November 2004; published online 7 January 2005)

\begin{abstract}
Hydrogen ion implantation is conventionally used to initiate the transfer of $\mathrm{Si}$ thin layers onto $\mathrm{Si}$ wafers coated with thermal oxide. In this work, we studied the feasibility of using plasma hydrogenation to replace high dose $\mathrm{H}$ implantation for layer transfer. Boron ion implantation was used to introduce H-trapping centers into $\mathrm{Si}$ wafers to illustrate the idea. Instead of the widely recognized interactions between boron and hydrogen atoms, this study showed that lattice damage, i.e., dangling bonds, traps $\mathrm{H}$ atoms and can lead to surface blistering during hydrogenation or upon postannealing at higher temperature. The $\mathrm{B}$ implantation and subsequent processes control the uniformity of $\mathrm{H}$ trapping and the trap depths. While the trap centers were introduced by $\mathrm{B}$ implantation in this study, there are many other means to do the same without implantation. Our results suggest an innovative way to achieve high quality transfer of $\mathrm{Si}$ layers without $\mathrm{H}$ implantation at high energies and high doses. () 2005 American Institute of Physics. [DOI: $10.1063 / 1.1852087$ ]
\end{abstract}

In recent years, an innovative approach for layer transfers, commonly known as ion-cut or smart-cut@, has attracted much attention. ${ }^{1}$ This process is based on high dose (typically $5 \times 10^{16} \mathrm{~cm}^{-2}$ ) hydrogen ion implantation and wafer bonding technologies. The crystalline quality of the transferred layer often suffers from hydrogen implantation damage, especially when very low implant energy is required for ultrathin layer transfers. ${ }^{2}$ Furthermore, the use of high dose hydrogen implantation results in process inefficiency and high cost. ${ }^{3,4}$ To improve the technology, preliminary investigations of the use of plasma hydrogenation to replace $\mathrm{H}$ implantation have been reported. ${ }^{5,6}$ However, the physical mechanisms of the hydrogenation process for layer transfers have yet to be elucidated to date.

Our objective was to investigate the feasibility of using plasma hydrogenation for Si layer transfer and to examine the H-trapping mechanisms under various processing conditions. N-type $<111>\mathrm{Si}$ wafers with a resistivity of $2-5$ $\Omega \mathrm{cm}$ were used. Boron was implanted at room temperature with an energy of $80 \mathrm{keV}$ and at three different doses of 1 $\times 10^{14}, 1 \times 10^{15}$, and $5 \times 10^{15} \mathrm{~cm}^{-2}$, respectively. For each

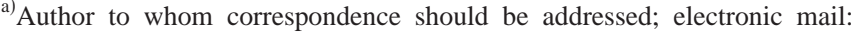
paul.chu@cityu.edu.hk
}

implant, part of the wafer was kept in the as-implanted state and the other part was electrically activated by annealing in vacuum $\left(\sim 3 \times 10^{-6} \mathrm{Torr}\right)$ at $900{ }^{\circ} \mathrm{C}$ for $15 \mathrm{~min}$. The $<111>$ orientation was selected here since hydrogen platelets prefer to form on the $\mathrm{Si}<111>$ planes under hydrogenation. ${ }^{7}$ Plasma hydrogenation was done in a plasma ion immersion implantation equipment using a rf plasma source. $^{8,9}$ The sample holder was negatively biased with a voltage of $500 \mathrm{~V}$ and kept at about 280 or $350{ }^{\circ} \mathrm{C}$ during hydrogenation for different time. Optical microscopy using Nomarski lenses was used to detect bubble formation on the sample surface after hydrogenation. The microscopic results were all based on observation at three different regions of the sample. Rutherford backscattering spectrometry in the channeling mode $(\mathrm{RBS} / \mathrm{C})$, elastic recoil detection (ERD), and cross-sectional transmission electron microscopy (XTEM) were used to characterize the samples before and after hydrogenation.

Nucleation of microbubbles is essential for thermal exfoliation of surface layers. Observation of surface blistering is a convenient way to gain insight into the mechanism of hydrogen-induced layer exfoliation. Samples implanted with boron with doses of $1 \times 10^{15}$ and $5 \times 10^{15} \mathrm{~cm}^{-2}$ showed surface bubbles after hydrogenation for $1.5 \mathrm{~h}$ at $350{ }^{\circ} \mathrm{C}$. The 
TABLE I. Summary of surface blistering results for activated and unactivated samples implanted with three different boron doses. The onset time, that is, time when surface blistering begins to emerge, is also shown here for two hydrogenation temperatures.

\begin{tabular}{|c|c|c|c|c|c|}
\hline \multirow{2}{*}{$\begin{array}{c}\text { Sample } \\
\text { No. }\end{array}$} & \multirow{2}{*}{$\begin{array}{l}\text { B-dose } \\
\left(\mathrm{cm}^{-2}\right)\end{array}$} & \multirow[b]{2}{*}{ Activation } & \multirow{2}{*}{$\begin{array}{c}\text { Bubble } \\
\text { density after } \\
\text { hydrogenation } \\
\text { at } 350{ }^{\circ} \mathrm{C} \\
\text { for } 1.5 \mathrm{~h} \\
\left(/ 10^{2} \mu \mathrm{m}^{2}\right)\end{array}$} & \multicolumn{2}{|c|}{$\begin{array}{c}\text { Onset time }(\mathrm{min}) \\
\text { (minimum time to cause surface blistering) } \\
\left({ }^{\circ} \mathrm{C}\right)\end{array}$} \\
\hline & & & & 350 & 280 \\
\hline 1 & $1 \times 10^{14}$ & No & 0 & \multicolumn{2}{|c|}{ N/A } \\
\hline 1a & $1 \times 10^{14}$ & Yes & 0 & \multicolumn{2}{|c|}{$\mathrm{N} / \mathrm{A}$} \\
\hline 2 & $1 \times 10^{15}$ & No & 9.0 & 60 & 240 \\
\hline $2 \mathrm{a}$ & $1 \times 10^{15}$ & Yes & 11 & 20 & 40 \\
\hline 3 & $5 \times 10^{15}$ & No & 8.1 & 15 & 30 \\
\hline $3 a$ & $5 \times 10^{15}$ & Yes & 13.1 & 5 & 10 \\
\hline
\end{tabular}

virgin $\mathrm{Si}$ control samples and samples implanted with the lowest dose of $1 \times 10^{14} \mathrm{~cm}^{-2}$ did not show any surface blistering at the same temperature for the same time or even much longer time up to $5 \mathrm{~h}$. The results are summarized in Table I. It should be pointed out that different hydrogenation time is required to cause surface blistering at different hydrogenation temperature, which is shown as onset time in Table I. A higher temperature naturally results in a shorter onset time and a higher boron implant dose leads to shorter time at the same hydrogenation temperature. It is also noted that for the same hydrogenation time, activation of boron before hydrogenation can enhance surface blistering, especially for the high dose sample.

Figure 1 shows the ion channeling results acquired from the boron implanted samples before hydrogenation, Nos. 2, 2a, 3, and 3a as described in Table I. For the as-implanted sample with a medium $B$ dose of $1 \times 10^{15} \mathrm{~cm}^{-2}$, a broad damage region (between channels 330 and 375) was observed. This broad damage peak is more prominent in the high $\mathrm{B}$ dose $\left(5 \times 10^{15} \mathrm{~cm}^{-2}\right)$ sample. After activation, the implant damage was generally annealed out in the near surface region, especially for the low and medium B dose samples. However, a significant increase in dechanneling occurred for the high dose sample around channel 340, indicat-

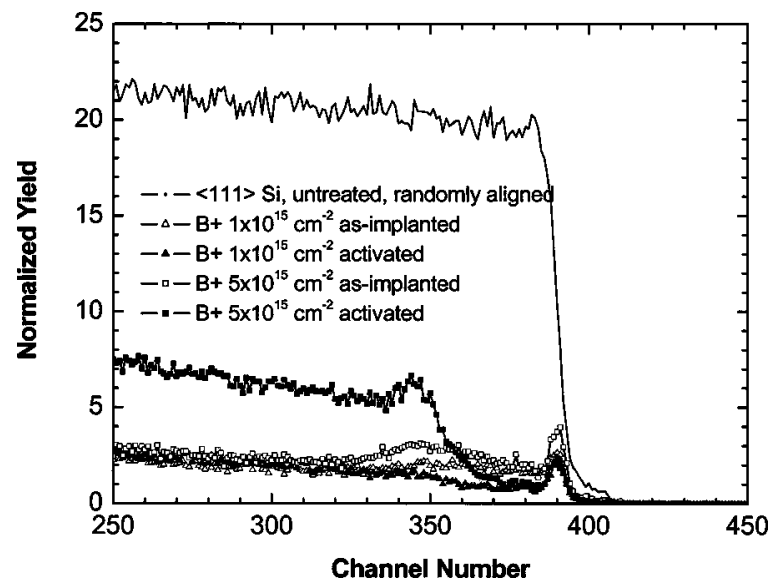

FIG. 1. RBS channeling results for sample Nos. 2, 2a, 3, and 3a described in Table I, before hydrogenation. The spectra were obtained with a $2.0 \mathrm{MeV}$ helium ion beam and a detector located at 13 deg from the incident beam. The helium beam was aligned along the $<111>$ axial direction. Also shown for comparison are a random spectrum obtained from the untreated silicon

annealing. Bright-field images, with 220 Bragg condition satisfied.
Downloaded 21 Dec 2006 to 134.94.122.39. Redistribution subject to AIP license or copyright, see http://apl.aip.org/apl/copyright ing coalescence of the ion implantation damage in this sample. In this sample, the peak B concentration $(\sim 1.9$ $\left.\times 10^{22} \mathrm{~cm}^{-3}\right)$ located at the projected range $R_{p}(\sim 260 \mathrm{~nm}$ below the surface) exceeds the solid solubility of boron in silicon at $900{ }^{\circ} \mathrm{C}\left(\sim 4.5 \times 10^{19} \mathrm{~cm}^{-3}\right){ }^{10}$ Not all B atoms can go into substitutional sites, leaving the nonsubstitutional atoms to form small boron clusters and other defects, which cause significant de-channeling in the channeling spectra. ${ }^{11}$ The ERD spectra (not shown here) indicate that the amount of hydrogen trapped in the high $\mathrm{B}$ dose $\left(5 \times 10^{15} \mathrm{~cm}^{-2}\right)$ samples with and without activation was almost equal. This result suggests that $\mathrm{H}$ trapping does not require substitutional or electrically activated B atoms. Simulation of the spectra with the computer code RUMP ${ }^{12}$ reveals a Gaussian shaped $\mathrm{H}$-concentration depth distribution peaking at a depth of about $230 \mathrm{~nm}$ and the integrated $\mathrm{H}$ dose amounts to 7 $\times 10^{15} \mathrm{~cm}^{-2}$.

Figures 2 and 3 show XTEM images obtained from samples after hydrogenation for $1.5 \mathrm{~h}$ at $350{ }^{\circ} \mathrm{C}$ that have been preimplanted with $5 \times 10^{15} \mathrm{~cm}^{-2}$ and $1 \times 10^{15} \mathrm{~cm}^{-2}$ boron, respectively. For the as-implanted sample with a boron dose of $5 \times 10^{15} \mathrm{~cm}^{-2}$, considerable damage and microcracks can be seen in the near surface region extending into the substrate [see Fig. 2(a)]. Trapping of $\mathrm{H}$ can occur at the defects extending from the surface to the projected range of the $\mathrm{B}$ implant as $\mathrm{H}$ diffuses into the Si. For the activated high-dose B sample [Fig. 2(b)], both the TEM and the channeling data show a greatly enhanced coalescence of implant damage, resulting in a very defective region near $R_{p}$. Long
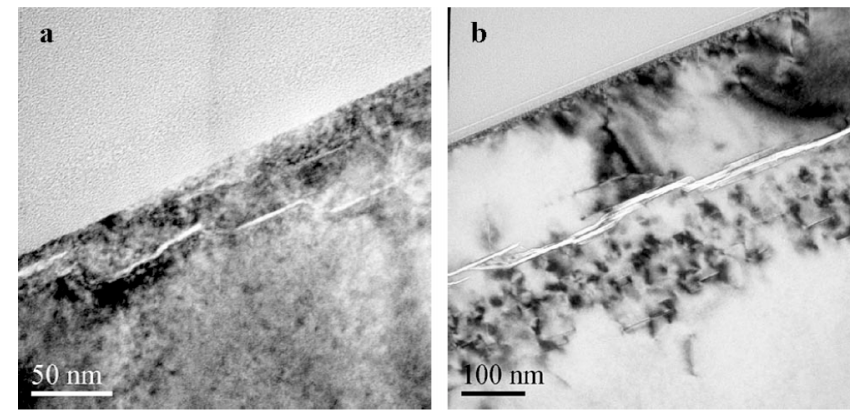

FIG. 2. Cross-section transmission electron micrographs obtained from the high $\mathrm{B}$ dose $\left(5 \times 10^{15} \mathrm{~cm}^{-2}\right)$ preimplanted sample after hydrogenation for $1.5 \mathrm{~h}$ at $350{ }^{\circ} \mathrm{C}$ : (a) without activation annealing, and (b) with activation annealing. Bright-field images, with 220 Bragg condition satisfied. 

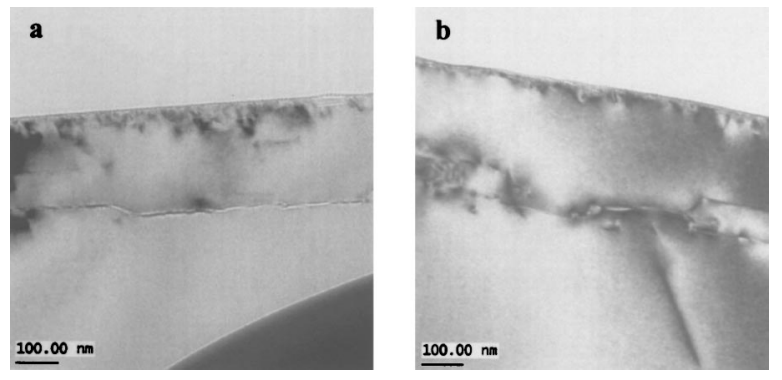

FIG. 3. Cross-sectional transmission electron micrographs obtained from the medium $\mathrm{B}$ dose $\left(1 \times 10^{15} \mathrm{~cm}^{-2}\right)$ preimplanted sample after hydrogenation for $1.5 \mathrm{~h}$ at $350{ }^{\circ} \mathrm{C}$ : (a) without activation annealing, and (b) with activation annealing. Bright-field images, with 220 Bragg condition satisfied.

microcracks are observed, located at a well-fined depth slightly above the very defective region. For the sample implanted with a B dose of $1 \times 10^{15} \mathrm{~cm}^{-2}$, both the asimplanted [Fig. 3(a)] and activated [Fig. 3(b)] samples exhibit very few defects other than the long hydrogen microcracks located at a well-defined depth, parallel to the surface. For the lowest B-dose sample $\left(1 \times 10^{14} \mathrm{~cm}^{-2}\right)$, a small amount of damage was observed by TEM and ion channeling before activation, and almost none after activation. No surface blistering or microcracks were observed in these samples after hydrogenation.

In order to replace $\mathrm{H}$ implantation with the hydrogenation process, the key issue is to enable hydrogen to diffuse into the substrate until the diffusing atoms get trapped at the desired depth for subsequent layer transfer. Therefore, it is critical to introduce trap sites at a well-defined depth, just below the desired depth for ion cutting, without deteriorating the crystalline quality of the layers above. The temperature for hydrogenation is also an important parameter. The structure of hydrogen-related defects created during hydrogenation is a strong function of hydrogenation temperature. 13,14 At relatively low temperatures (e.g., $<250{ }^{\circ} \mathrm{C}$ ), hydrogen platelets form in the near surface region, within $100 \mathrm{~nm}$ of the surface. However, platelet nucleation is suppressed at temperatures above $250^{\circ} \mathrm{C}$. To avoid platelet nucleation in the near surface region, we set the hydrogenation temperature at around $350{ }^{\circ} \mathrm{C}$.

In the case of ion cutting, Höchbauer et al. ${ }^{15}$ have shown that the ion-cut depth in $\mathrm{Si}$ is largely controlled by lattice damage induced by the hydrogen implantation. In the case of hydrogenation, the results show that the trap centers for the hydrogen atoms originate from lattice defects (e.g., dangling bonds) introduced by boron implantation. The boron implantation conditions and subsequent annealing processes thus control the uniformity of $\mathrm{H}$ trapping and the depths where $\mathrm{H}$ will be trapped. In the case of the medium dose boron implant $\left(1 \times 10^{15} \mathrm{~cm}^{-2}\right)$, the sample retained good crystalline quality after implantation except in the near surface region. Upon hydrogenation, $\mathrm{H}$ atoms were able to diffuse into the $\mathrm{Si}$ and get trapped at the depth where the boron implantinduced defects reside, as observed for samples without activation. The diffusion and trapping of $\mathrm{H}$ lead to the formation of microcracks located at a well-defined depth. In this study, the medium B dose of $1 \times 10^{15} \mathrm{~cm}^{-2}$ without activation appears to be an appropriate processing condition for hydrogenation, except for some damage in the very near surface region that may require certain surface processing before wafer bonding. It should be noted that activation of the B implant in this sample essentially led to the same results. In the as-implanted high B dose sample $\left(5 \times 10^{15} \mathrm{~cm}^{-2}\right), \mathrm{H}$ traps were generally present, extending from the near surface region to the projected range of the B implantation, causing microcracks to form throughout an extended depth region. In the activated high $\mathrm{B}$ dose sample, ion implantation damage was annealed out in the near-surface region accompanied by significant coalescence of implant damage located near the projected range, thus resulting in a uniform trap region for $\mathrm{H}$ atoms at a specific depth.

In summary, we have investigated the use of plasma hydrogenation for layer transfers. In the present study, hydrogen atoms introduced by plasma hydrogenation are found to be trapped by lattice damage (e.g., dangling bonds) induced by boron preimplantation. The trapped $\mathrm{H}$ atoms cause surface blistering. Boron implantation with a dose of 1 $\times 10^{15} \mathrm{~cm}^{-2}$, with or without activation, can provide sufficient trap sites at a well-defined depth for trapping of hydrogen during hydrogenation. The preimplantation and subsequent annealing processes control the uniformity of $\mathrm{H}$ traps and the trapped depths. While the trap centers were introduced by B implantation in the present study, there are many other means to do the same without such implantation. Our study suggests a possible process of layer transfer, without the use of ion implantation of hydrogen or any other gaseous element, with potential applications for the formation of silicon-on-insulator and other related structures.

The work was jointly supported by Hong Kong Research Grants Council (RGC) Competitive Earmarked Research Grant (CERG) 1137/03E, and City University of Hong Kong Strategic Research Grant (SRG) 7001642. UCSD and ASU gratefully acknowledge sponsorship from NSF. The work at Los Alamos National Laboratory was supported by the DOE Office of Basic Energy Sciences.

${ }^{1}$ M. Bruel, Electron. Lett. 31, 1201 (1995).

${ }^{2}$ C. Qian, B. Terreault, and S. C. Gujrathi, Nucl. Instrum. Methods Phys. Res. B 175-177, 711 (2001).

${ }^{3}$ K. Henttinen, I. Suni, and S. S. Lau, Appl. Phys. Lett. 76, 2370 (2000).

${ }^{4}$ Y. Zheng, S. S. Lau, T. Hochbauer, A. Misra, R. Verda, X.-M. He, M. Nastasi, and J. W. Mayer, J. Appl. Phys. 89, 2972 (2001).

${ }^{5}$ A. Y. Usenko and A. G. Ulyashin, Jpn. J. Appl. Phys., Part 1 41, 5021 (2002).

${ }^{6}$ A. Usenko, J. Electron. Mater. 32, 872 (2003).

${ }^{7}$ F. A. Reboredo, M. Ferconi, and S. T. Pantelides, Phys. Rev. Lett. 82, 4870 (1999).

${ }^{8}$ P. K. Chu, S. Qin, C. Chan, N. W. Cheung, and L. A. Larson, Mater. Sci. Eng., R. 17, 207 (1996).

${ }^{9}$ P. K. Chu, B. Y. Tang, Y. C. Cheng, and P. K. Ko, Rev. Sci. Instrum. 68, 1866 (1997).

${ }^{10}$ B. Garben, W. A. Orr-Arienzo, and R. F. Lever, J. Electrochem. Soc. 133, 2152 (1986).

${ }^{11}$ J. R. Liefting, R. J. Schreutelkamp, J. Vanhellemont, W. Vandervorst, K. Maex, J. S. Custer, and F. W. Saris, Appl. Phys. Lett. 63, 1134 (1993).

${ }^{12}$ L. R. Doolittle, Nucl. Instrum. Methods Phys. Res. B 9, 344 (1985).

${ }^{13}$ N. M. Johnson, F. A. Ponce, R. A. Street, and R. J. Nemanich, Phys. Rev. B 35, 4166 (1987).

${ }^{14}$ N. H. Nickel, G. B. Anderson, N. M. Johnson, and J. Walker, Phys. Rev. B 62, 8012 (2000).

${ }^{15}$ T. Höchbauer, A. Misra, M. Nastasi, and J. W. Mayer, J. Appl. Phys. 92, 2335 (2002). 